\title{
ENERGY STABILITY OF THE MUSCL SCHEME
}

\author{
Qaisar Abbas $^{*, 1}$, Edwin van der Weide ${ }^{\dagger}$ and Jan Nordström* ${ }^{*,+, 2}$ \\ *Department of Information Technology, Scientific Computing \\ Uppsala University, SE-751 05 Uppsala, Sweden, \\ ${ }^{1}$ qaisar.abbas@it.uu.se. \\ ${ }^{\dagger}$ Faculty of Engineering Technology \\ University of Twente, 7500 AE Enschede, The Netherlands, \\ vdweide@gmail.com. \\ $\ddagger$ School of Mechanical, Industrial and Aeronautical Engineering \\ University of the Witvatersrand, PO WITS 2050, Johannesburg, South Africa, and \\ Department of Aeronautics and Systems Integration \\ FOI, The Swedish Defence Research Agency, SE-164 90 Stockholm, Sweden, \\ 2 jan.nordstrom@it.uu.se.
}

Keywords: MUSCL Scheme, Energy Estimates and Numerical Stability, Summation-by-parts Form, Artificial Dissipation

\begin{abstract}
We analyze the energy stability of the standard MUSCL scheme. The analysis is possible by reformulating the MUSCL scheme in the framework of summation-by-parts (SBP) operators including an artificial dissipation. The effect of different slope limiters is studied. It is found that all the slope limiters do not lead to the correct sign of the entries in the dissipation matrix. The implication of that is discussed for both linear and nonlinear scalar problems.
\end{abstract}

\section{Introduction}

For problems involving shocks which arise in computational fluid mechanics and related areas, the MUSCL scheme [1] is a very effective approach to resolve discontinuities. This scheme ensures the monotonicity of the solution for the whole computational time and it is arguably computationally less expensive compared to relevant counterparts like the WENO schemes [2] for approximately the same accuracy.

In this paper, we have reformulated the MUSCL scheme in summation-by-parts (SBP) form including an artificial dissipation operator. Related work can be found in [3, 4], where the WENO scheme has been formulated in a similar way. The SBP operators are well-established theoretically [9] and their usefulness is proven for practical applications, see [10, 11, 12].

In this work we will investigate the MUSCL scheme to see if the scheme is energy stable, i.e. stable in the $L_{2}$-norm, see $[3,4]$. We consider both scalar linear and nonlinear hyperbolic problems in one dimension. Our analysis is based on theoretical as well as numerical observations. 


\section{The MUSCL Scheme in SBP Form}

Consider the unsteady one-dimensional conservation law

$$
u_{t}+f(u)_{x}=0, \quad 0 \leq x \leq 1, \quad t \geq 0
$$

Define a uniform grid $x_{j}=j \Delta x, j=0, \ldots, N$, with $\Delta x=1 / N$. On the grid, define a flux $F(U)$, where $U=\left[U_{0}(t), U_{1}(t) \ldots, U_{N}(t)\right]^{T}$ is the discrete approximation of the solution $u$ in Eq. (1). The second order upwind discretization of Eq. (1) using the MUSCL approach [1] results in

$$
U_{t}+R E S_{i}=0, \quad R E S_{i}=\frac{1}{\Delta x}\left(F_{i+\frac{1}{2}}-F_{i-\frac{1}{2}}\right) .
$$

In Eq. (2), $F_{i+\frac{1}{2}}$ is the flux function at the interface $i+\frac{1}{2}$. More details on the computation of numerical flux function can be found in [5].

Similarly a second order discretization of the flux function in Eq. (1), obeying the SBP property [9] and with the introduction of artificial dissipation on SBP form [6] leads to

$$
U_{t}+D_{2} F=-P^{-1} \tilde{D}_{1}^{T} B_{M} \tilde{D}_{1} U
$$

where $D_{2}$ is the central finite difference operator on SBP form given by

$$
\begin{gathered}
D_{2}=P^{-1} Q, \quad Q+Q^{T}=B, \quad P=P^{T}, \\
D_{2}=\frac{1}{2 \Delta x}\left[\begin{array}{ccccc}
-2 & 2 & & & \\
-1 & 0 & 1 & & \\
& \ddots & \ddots & \ddots & \\
& & -1 & 0 & 1 \\
& & & -2 & 2
\end{array}\right], \quad P^{-1}=\frac{1}{\Delta x}\left[\begin{array}{ccccc}
2 & & & \\
& 1 & & & \\
& & \ddots & & \\
& & 1 & \\
& & & 2
\end{array}\right],
\end{gathered}
$$

and $B=\operatorname{diag}(-1,0, \ldots, 0,1)$. The term $-P^{-1} \tilde{D}_{1}^{T} B_{M} \tilde{D}_{1} U$ in Eq. (3) is an artificial dissipation operator. It will be shown below that the matrix $B_{M}$ can be constructed such that Eq. (3) corresponds to the standard second order MUSCL formulation [1] which means that the formulations given by Eq. (2) and Eq. (3) are equivalent. $\widetilde{D}_{1}$ is a two point difference operator and the matrix $B_{M}$ is a diagonal matrix, see Eq. (5).

$$
\widetilde{D}_{1}=\left[\begin{array}{ccccc}
-1 & 1 & & & \\
& -1 & 1 & & \\
& & \ddots & \ddots & \\
& & & -1 & 1 \\
& & & -1 & 1
\end{array}\right], \quad B_{M}=\left[\begin{array}{ccccc}
b_{0} & & & & 0 \\
& b_{1} & & & \\
& & \ddots & & \\
& & & b_{N-1} & \\
0 & & & & 0
\end{array}\right]
$$

\subsection{Explicit Form of $B_{M}$}

At an interior point $i$, we have

$$
\left\{-P^{-1} \widetilde{D}_{1}^{T} B_{M} \widetilde{D}_{1} U\right\}_{i}=-\frac{1}{\Delta x}\left(b_{i-1} \Delta U_{i-1}-b_{i} \Delta U_{i}\right),
$$


where $\Delta U_{i}=U_{i+1}-U_{i}$. In combination with the central discretization of the convective term, this leads to the following formulation of the residual for an internal node

$$
\Delta x R E S_{i}=\frac{1}{2}\left(F_{i+1}-F_{i-1}\right)+b_{i-1} \Delta U_{i-1}-b_{i} \Delta U_{i} .
$$

For the boundary nodes $x_{0}$ and $x_{N}$, the residuals are

$$
\Delta x R E S_{0}=\Delta F_{0}-\widetilde{P}_{0}^{-1} b_{0} \Delta U_{0}, \quad \Delta x R E S_{N}=\Delta F_{N-1}+\widetilde{P}_{N}^{-1} b_{N-1} \Delta U_{N-1},
$$

where $\widetilde{P}_{0}^{-1}=\widetilde{P}_{N}^{-1}=2$. Comparing Eqs. (2) and (7), it is clear that both schemes are identical if

$$
b_{i} \Delta U_{i}=\frac{1}{2}\left(F_{i+1}+F_{i}\right)-F_{i+\frac{1}{2}} .
$$

It can be shown that the $b_{i}$ in Eq. (9) becomes

$$
b_{i}=\frac{1}{2}\left\{\left|A_{i+\frac{1}{2}}\right|\left(1-\frac{\phi_{i}}{2}-\frac{\psi_{i+1}}{2}\right)+A_{R_{i+\frac{1}{2}, i+1}} \frac{\psi_{i+1}}{2}-A_{L_{i+\frac{1}{2}, i}} \frac{\phi_{i}}{2}\right\},
$$

where $\phi_{i}$ and $\psi_{i+1}$ are the slope limiters involved in the fluxes. They are related in the following way.

$$
\phi_{i}=\phi\left(r_{i}\right)=\phi\left(\frac{\Delta U_{i-1}}{\Delta U_{i}}\right)=\frac{\Delta U_{i-1}}{\Delta U_{i}} \phi\left(\frac{1}{r_{i}}\right)=\frac{\Delta U_{i-1}}{\Delta U_{i}} \psi_{i}
$$

Also $A=\frac{\partial F}{\partial U}$ is a Jacobian matrix evaluated at the Roe average states. The property of a Roe average state is that $f_{2}-f_{1}=A_{R o e}\left(u_{2}-u_{1}\right)$.

\subsection{Energy Stability}

In this section we define the two versions of the energy stability, that we will work with in the analysis below.

Definition. Consider Eq. (3) and Eq. (12). The scheme defined by Eq. (3) is pointwise energy stable if $b_{i} \geq 0$ for all $i=0,1 \ldots, N$. The scheme defined by Eq. (3) is energy stable in the mean if $(D U)^{T} B_{M}(D U) \geq 0$, where $D U=\left[(D U)_{0},(D U)_{1}, \ldots,(D U)_{N}\right]^{T}$.

Remark. Pointwise energy stable schemes lead to energy stable schemes in the mean. The reverse is not true.

\subsection{Energy Estimates}

To investigate whether the scheme defined in Eq. (3) is energy stable or not, we start by considering the linear constant coefficient case with $F=a U$ and use the energy method. Multiplying Eq. (3) with $U^{T} P$, adding its transpose and using Eq. (4) leads to

$$
\frac{d}{d t}\|U\|_{P}^{2}+a U^{T} B U=-2\left(\widetilde{D}_{1} U\right)^{T} B_{M}\left(\widetilde{D}_{1} U\right) .
$$

where $\|U\|_{P}^{2}=U^{T} P U$. For a bounded solution and energy stability we must have $\frac{d}{d t}\|U\|_{P}^{2} \leq$ 0 . The boundary terms $U^{T} B U=U_{0}^{2}-U_{N}^{2}$ can be bounded using the SAT boundary treatment 
[8] and are ignored from now on. The right-hand side of Eq. (12) is negative if the matrix $B_{M}$ is positive semi-definite. The matrix $B_{M}$ for a linear problem becomes

$$
b_{i}=\frac{1}{2}\left\{|A|-\phi_{i} A^{+}+\psi_{i+1} A^{-}\right\}
$$

where $A^{+}$contains the positive eigenvalues of $A$ and $A^{-}$the negative ones,

$$
A^{+}=\frac{1}{2}(A+|A|), \quad A^{-}=\frac{1}{2}(A-|A|) .
$$

For a scalar problem with $F=a U$, Eq. (13) reduces to

$$
b_{i}=\frac{1}{2} a\left\{1-\phi_{i}\right\}, \quad a>0, \quad \text { and } \quad b_{i}=\frac{1}{2}|a|\left\{1-\psi_{i+1}\right\}, \quad a<0 .
$$

From the theory of the slope limiters [7] we have that $0 \leq \phi_{i}, \psi_{i+1} \leq 2$. It is obvious that any limiter which takes values greater than 1 , will lead to $b_{i} \leq 0$ in the computational domain and hence no pointwise energy stability. In $[3,4]$, the authors modified the WENO scheme by correcting this anomaly of the scheme. We will discuss below whether that is necessary and meaningful.

\section{Numerical Results}

Consider Eqs. (10), (11) and (12). It is obvious that the sign of $b_{i}$ depends on the slope limiters involved in the MUSCL scheme. If the solution is smooth, we have $\phi_{i}=\psi_{i+1}=1$, and for all $A, b_{i}$ will be zero. For problems with discontinuities, we could have $0 \leq \phi_{i}, \psi_{i+1} \leq 2$, which decides the sign of $b_{i}$ in non-smooth regions.

We consider a linear problem $(f=u)$ first with a step discontinuity as initial data and analyze four different limiters. All the results are shown for $N=80$ and $t=0.3$. In Figures 1-4 we have shown the minimum of $b_{i}$ and $-\left(D_{1} U\right)^{T} B_{M}\left(D_{1} U\right)$ at each time step for minmod, VanLeer, superbee and MC limiters. The minmod limiter have $b_{i} \geq 0$ for all time and hence is pointwise stable. All other limiters lead to $b_{i}<0$ at few points near the discontinuity. It means that these limiters do not lead to pointwise stability. It is also found that $-\left(D_{1} U\right)^{T} B_{M}\left(D_{1} U\right) \leq$ 0 for all limiters for the whole computational time which gives energy stability in the mean.

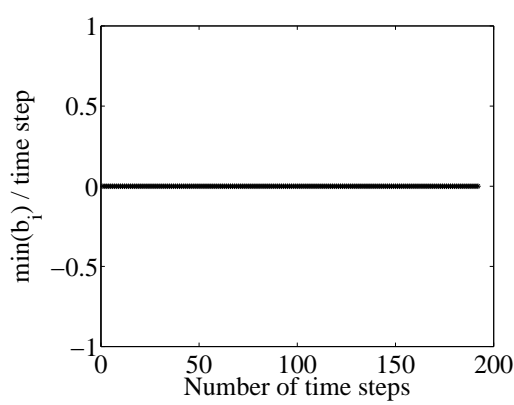

(a) $\min \left(b_{i}\right)$ per time step

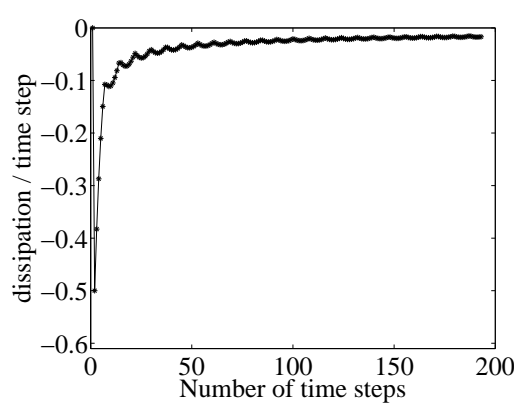

(b) $-\left(D_{1} U\right)^{T} B_{M}\left(D_{1} U\right)$

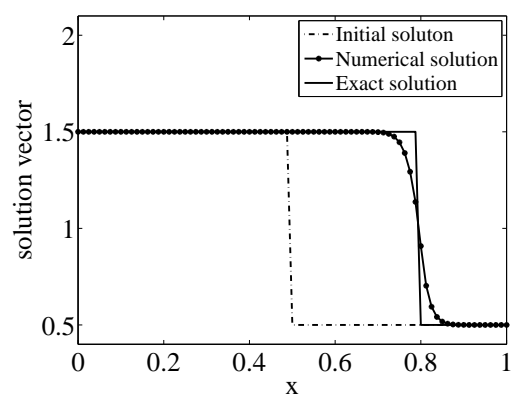

(c) solution, $N=80, t=0.3$

Figure 1: Results from the MUSCL in SBP form using the minmod limiter, $f=u$.

Next we consider the Burger equation with $f=\frac{u^{2}}{2}$ in Eq. (1) and repeat the same analysis with the minmod, VanLeer and MC limiters. It is found that all the tested limiters have some 


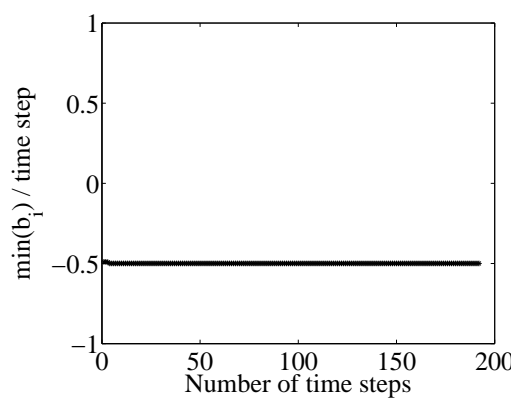

(a) $\min \left(b_{i}\right)$ per time step

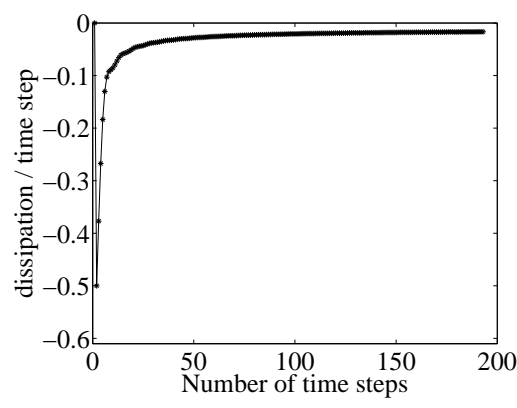

(b) $-\left(D_{1} U\right)^{T} B_{M}\left(D_{1} U\right)$

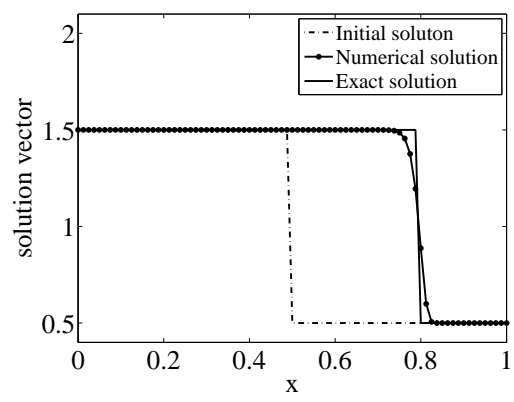

(c) solution, $N=80, t=0.3$

Figure 2: Results from the MUSCL in SBP form using the Van Leer limiter, $f=u$.

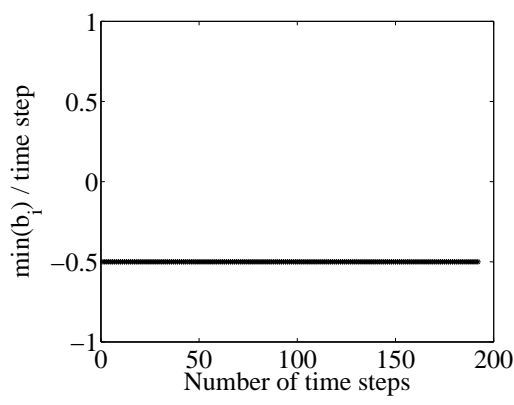

(a) $\min \left(b_{i}\right)$ per time step

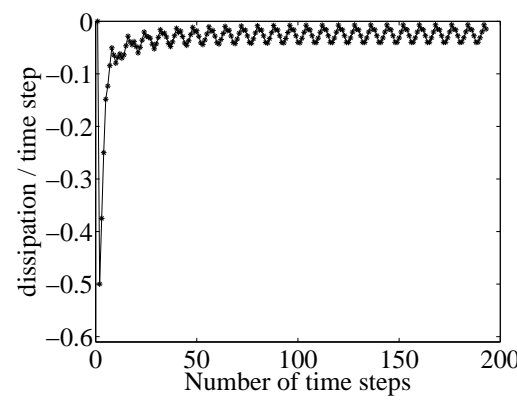

(b) $-\left(D_{1} U\right)^{T} B_{M}\left(D_{1} U\right)$

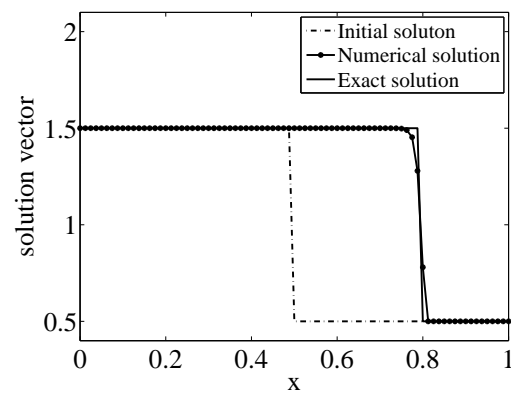

(c) solution, $N=80, t=0.3$

Figure 3: Results from the MUSCL in SBP form using the Superbee limiter, $f=u$.

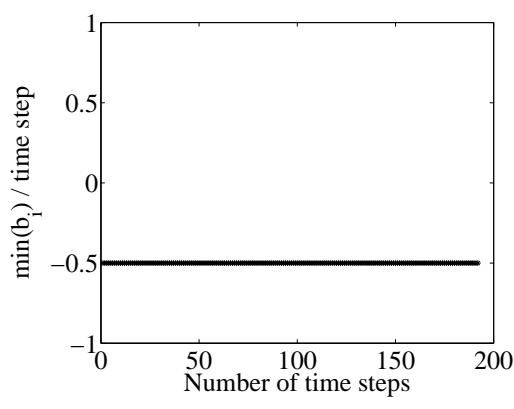

(a) $\min \left(b_{i}\right)$ per time step

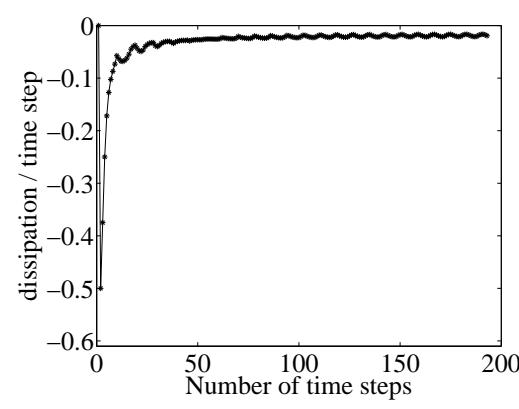

(b) $-\left(D_{1} U\right)^{T} B_{M}\left(D_{1} U\right)$

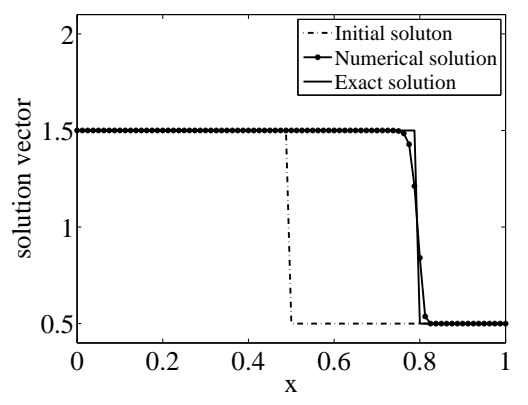

(c) solution, $N=80, t=0.3$

Figure 4: Results from the MUSCL in SBP form using the MC limiter, $f=u$.

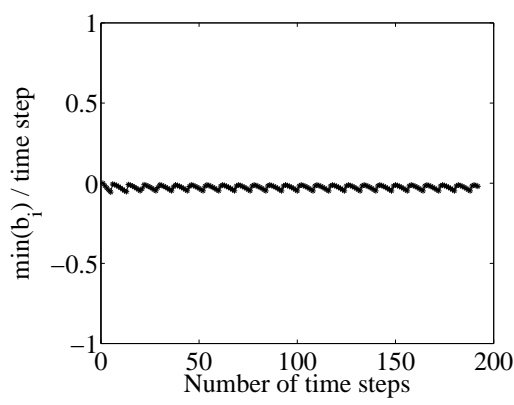

(a) $\min \left(b_{i}\right)$ per time step

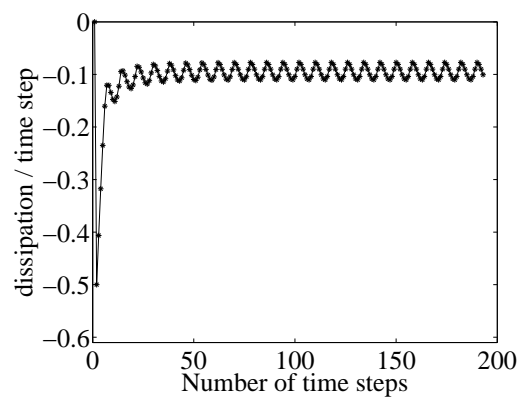

(b) $-\left(D_{1} U\right)^{T} B_{M}\left(D_{1} U\right)$

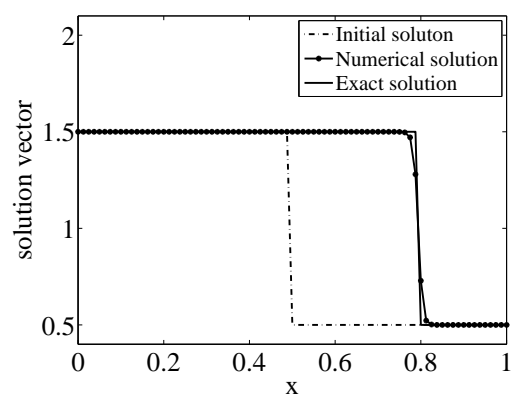

(c) solution, $N=80, t=0.3$

Figure 5: Results from the MUSCL in SBP form using the minmod limiter, $f=\frac{u^{2}}{2}$. 


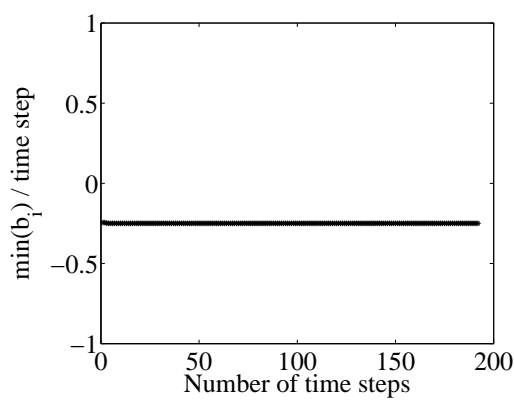

(a) $\min \left(b_{i}\right)$ per time step

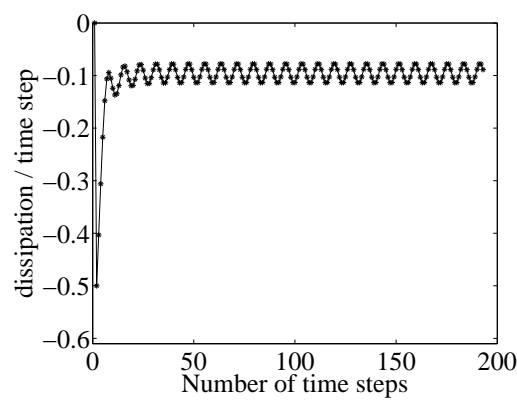

(b) $-\left(D_{1} U\right)^{T} B_{M}\left(D_{1} U\right)$

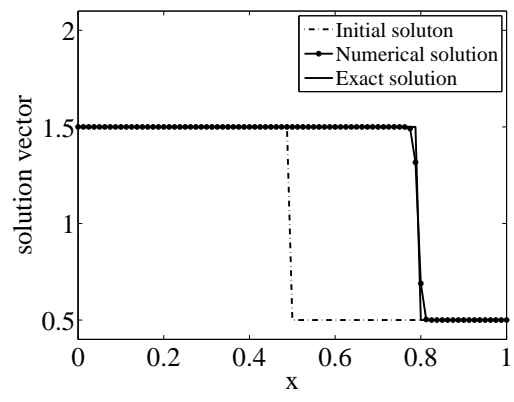

(c) solution, $N=80, t=0.3$

Figure 6: Results from the MUSCL in SBP form using the Van Leer limiter, $f=\frac{u^{2}}{2}$.

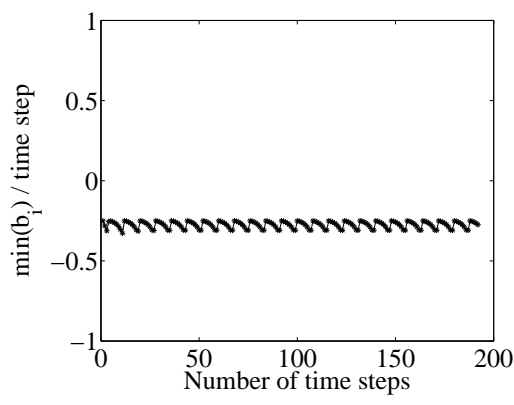

(a) $\min \left(b_{i}\right)$ per time step

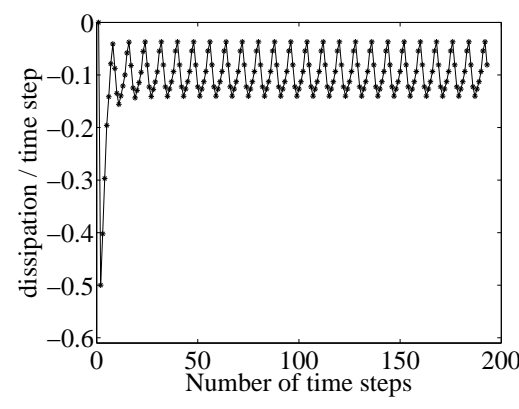

(b) $-\left(D_{1} U\right)^{T} B_{M}\left(D_{1} U\right)$

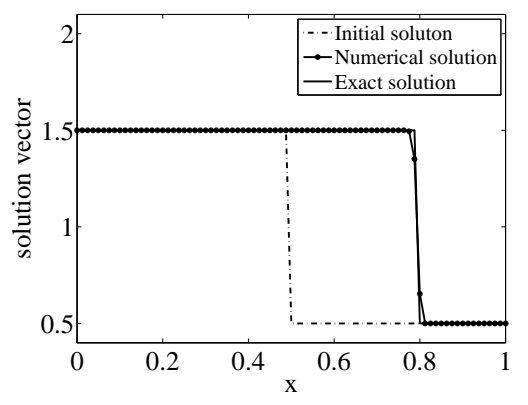

(c) solution, $N=80, t=0.3$

Figure 7: Results from the MUSCL in SBP form using the Superbee limiter, $f=\frac{u^{2}}{2}$.

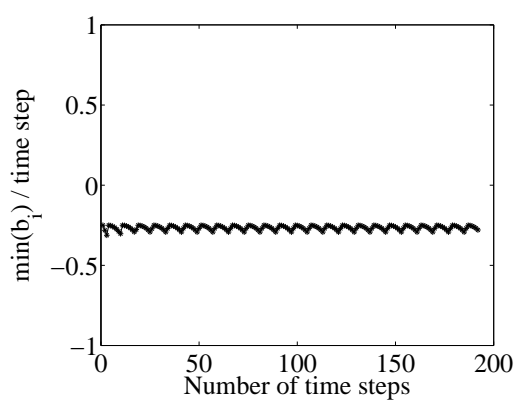

(a) $\min \left(b_{i}\right)$ per time step

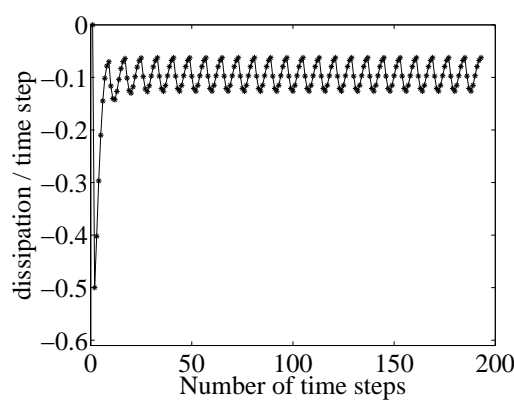

(b) $-\left(D_{1} U\right)^{T} B_{M}\left(D_{1} U\right)$

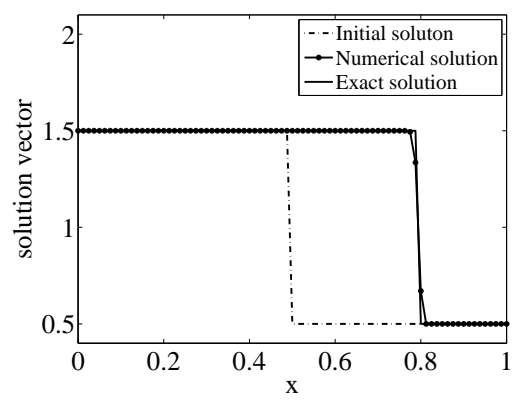

(c) solution, $N=80, t=0.3$

Figure 8: Results from the MUSCL in SBP form using the MC limiter, $f=\frac{u^{2}}{2}$.

$b_{i}<0$ but the minmod limiter is almost zero for all time leading to pointwise stability, see Figures 5-8. It can also be seen that all schemes are stable in the mean.

It is not clear whether pointwise stability is necessary or if stability in the mean is enough. If we replace $b_{i}<0$ in the matrix $B_{M}$ with $b_{i}=0$ at each time step, we find that it leads to an additional and excessive amount of dissipation in the discontinuity/shock region, see Table 1 for $l_{2}$-error of solutions. By demanding the pointwise stability, clearly the sharpness of the shock decreases. 
Table 1: Analysis of different limiters for the linear problem $(a=1, t=0.3)$

\begin{tabular}{lll}
\hline Limiters & $l_{2}$-error, $\min \left(b_{i} \leq 0\right)$ & $l_{2}$-error, $\min \left(b_{i}=0\right)$ \\
\hline minmod & 0.0711 & 0.0711 \\
Van Leer & 0.0578 & 0.0642 \\
Superbee & 0.0400 & 0.0634 \\
MC & 0.0504 & 0.0639 \\
\hline
\end{tabular}

\section{Conclusion}

We have expressed the MUSCL scheme as a combination of an SBP operator and an artificial dissipation operator. This form allows us to use the energy method for analyzing stability. Our main interest was to look at the behavior of dissipation matrix $B_{M}$ in Eq. (5), which is crucial for the stability of the scheme and also influence the sharpness of the shock.

As the matrix depends on the slope limiters of the MUSCL scheme, it was found most of the tested limiters except minmod limiter do not lead to pointwise stability while all limiters are stable in the mean.

By making the schemes pointwise stable by replacing $b_{i}<0$ in the matrix $B_{M}$ with $b_{i}=0$ resulted in an additional and excessive dissipation for all the limiters. It was shown that the error in the calculations increased and the sharpness of the shock decreased. This procedure was used in $[3,4]$ but seems questionable.

\section{References}

[1] B. van Leer. Towards the Ultimate Conservative Difference Scheme, V. A Second Order Sequel to Godunov's Method, J. Comput. Phys. 32:101-136, 1979.

[2] G. Jiang, and C.W. Shu. Efficient implementation of weighted ENO schemes, J. Comput. Phys.126:202-228, 1996.

[3] N.K. Yamaleev, and M.H. Carpenter. Third-order energy stable WENO scheme, J. Comput. Phys. 228:3025-3047, 2009.

[4] N.K. Yamaleev, and M.H. Carpenter. A systematic methodology for constructing highorder energy stable WENO schemes, J. Comput. Phys. 228:4248-4272, 2009.

[5] Q. Abbas, E. van der Weide, and J. Nordström. Accurate and stable calculations involving shocks using a new hybrid scheme. AIAA Paper No. 2009-3985, 2009.

[6] K. Mattsson, M. Svärd, and J. Nordström. Stable and Accurate Artificial Dissipation. J. Sci. Comput. 21(1):57-79, 2004.

[7] M. Berger, M.J. Aftosmis, and S.M. Murman. Analysis of slope limiters on irregular grids, 43rd AIAA Aerospace Sciences Maeeting, Jan 10-13, 2005.

[8] M. H. Carpenter, D. Gottlieb, and S. Abarbanel, The stability of numerical boundary treatments for compact high-order finite difference schemes, J. Comput. Phys. 108(2):272-295, 1994.

[9] M. H. Carpenter, J. Nordström, and D. Gottlieb, A Stable and Conservative Interface Treatment of Arbitrary Spatial Accuracy, J. Comput. Phys. 148(2):341-365, 1999. 
[10] M. Svärd, M. H. Carpenter, and J. Nordström, A Stable High-Order Finite Difference Scheme for the Compressible Navier-Stokes Equations, far-field boundary conditions, $J$. Comput. Phys. 225(1):1020-1038, 2007.

[11] M. Svärd, and J. Nordström, A Stable High-Order Finite Difference Scheme for the Compressible Navier-Stokes Equations: Wall Boundary Conditions, J. Comput. Phys. 227:4805-4824, 2008.

[12] J. Nordström, J. Gong, E. van der Weide, and M. Svärd, A Stable and Conservative High Order Multi-block Method for the Compressible Navier-Stokes Equations, J. Comput. Phys. 228:9020-9035, 2009. 